\title{
Nucleon structure functions and longitudinal spin asymmetries
}

\author{
Harleen Dahiya ${ }^{1, a}$, Monika Randhawa ${ }^{2}$, and Nisha Dhiman ${ }^{1}$ \\ ${ }^{1}$ Department of Physics,Dr. B.R. Ambedkar National Institute of Technology, Jalandhar, 144011, India \\ ${ }^{2}$ University Institute of Engineering and Technology, Panjab University, Chandigarh, 160014, India
}

\begin{abstract}
The chiral constituent quark model $(\chi \mathrm{CQM})$ has been used to analyse the phenomenological dependence of the spin independent $\left(F_{1}^{p, n}\right.$ and $\left.F_{2}^{p, n}\right)$ and the spin dependent $\left(g_{1}^{p, n}\right)$ structure functions of the nucleon on the the Bjorken scaling variable $x$. The implication of the presence of the sea quarks has been discussed for the $p$ and $n$ longitudinal spin asymmetries $\left(A_{1}^{p}(x)\right.$ and $\left.A_{1}^{n}(x)\right)$ ratio of polarized to unpolarized quark distribution functions for up and down quarks in the $p$ and $n \frac{\Delta u^{p}(x)}{u^{p}(x)}, \frac{\Delta d^{p}(x)}{d^{p}(x)}, \frac{\Delta u^{n}(x)}{u^{n}(x)}$, and $\frac{\Delta d^{n}(x)}{d^{n}(x)}$. The results have been compared with the recent available experimental observations.
\end{abstract}

\section{Introduction}

Several interesting studies have been carried out to understand the internal structure of the nucleon ever since the deep inelastic scattering (DIS) experiments revealed that the quarks are point-like constituents [1] identified as the valence or constituent quarks. Surprisingly, the measurements of polarized structure functions of proton in DIS experiments [2-5] showed that the total spin carried by the constituent quarks was very small (only about 30\%) leading to the "proton spin crisis" [6]. The polarized deep inelastic lepton-nucleon scattering is an useful probe of the spin structure of the nucleon and the measurements with proton, deuteron, and helium-3 targets have determined the unpolarized and polarized structure functions of the nucleon through the measurement of the longitudinal spin asymmetries with the target spin being parallel and antiparallel to the longitudinally polarized beam $[3,7]$. In addition to this, major surprise has been revealed in the famous DIS experiments by the New Muon Collaboration (NMC) [8], Fermilab E866 [9], Drell-Yan cross section ratios of the NA51 experiments [10] and more recently by HERMES [11]. These experiments established the violation of Gottfried sum rule (GSR) $\left(\int_{0}^{1}[\bar{d}(x)-\bar{u}(x)] d x\right)$ [12] confirming the sea quark asymmetry of the unpolarized quarks in the case of nucleon. Even though extensive studies have been carried out in the past 40 years but it is still a big challenge to perform the calculations from the first principles of Quantum Chromodynamics (QCD). Confinement has limited our knowledge on the composition of hadrons and internal structure continues to remain a major unresolved problem in high energy spin physics.

Recently, the neutrino-induced DIS experiments [13] have emphasized that the sea quarks dominate for the values of Bjorken scaling variable $x<0.3$ and precision data have been collected only in the low and moderate $x$ regions due to experimental limitation. Further, the experiments CDHS [14], CCFR [15], CHARMII [16], NOMAD [17], NuTeV [18] and CHORUS [19] have pointed out

\footnotetext{
ae-mail: dahiyah@nitj.ac.in
} 
the need for additional refined data renewing considerable interest in the non-valence structure. In the absence of precise data above $x>0.4$ which is a relatively clean region to test the valence structure of the nucleon, the parametrizations are quite unconstrained.

One of the most successful nonperturbative approach is the chiral constituent quark model $(\chi \mathrm{CQM})[20,21]$. The basic idea is based on the possibility that chiral symmetry breaking takes place at a distance scale much smaller than the confinement scale. The $\chi \mathrm{CQM}$ uses the effective interaction Lagrangian approach of the strong interactions where the effective degrees of freedom are the valence quarks and the internal Goldstone bosons (GBs) which are coupled to the valence quarks [22-25]. The $\chi \mathrm{CQM}$ successfully explains the spin structure of the nucleon [25], magnetic moments of octet and decuplet baryons [26], semileptonic weak decay parameters [27], magnetic moments of nucleon resonances and $\Lambda$ resonances [28], quadrupole moment and charge radii of octet baryons [29], etc.. On the other hand, the inclusion of Bjorken scaling variable $x$ in the distributions functions is obtained by fitting parametrizations to data.

\section{Unpolarized and polarized distribution functions of quarks}

The unpolarized distribution function of the quark (antiquark) $q_{i}(x)\left(\bar{q}_{i}(x)\right)$ is described as the probability of the $i^{\text {th }}$ quark (antiquark) carrying a fraction $x$ of the nucleon's momentum. It can be calculated from the scalar matrix element of the nucleon using the operator $q \bar{q}$ measuring the sum of the quark and antiquark numbers as $\langle N|q \bar{q}| N\rangle$, where $|N\rangle$ is the nucleon wavefunction. The operator $q \bar{q}$ is defined in terms of the number $n_{q(\bar{q})}$ of $q(\bar{q})$ quarks with electric charge $e_{q}\left(e_{\bar{q}}\right)$.

The polarized distribution function of the $i^{\text {th }}$ quark $\Delta q_{i}(x)$ is defined as $\Delta q_{i}(x)=q_{i}^{\uparrow}(x)-q_{i}^{\downarrow}(x)$, where $q_{i}^{\uparrow}(x)\left(q_{i}^{\downarrow}(x)\right)$ is the probability that the $i^{\text {th }}$ quark spin is aligned parallel or antiparallel to the nucleon spin. The polarized distribution function of the quarks can be calculated from the axial vector matrix element of the nucleon using the operator $q^{\uparrow} q^{\downarrow}$ measuring the sum of the quark with spin up and down as $\left\langle N\left|q^{\uparrow} q^{\downarrow}\right| N\right\rangle$. Here $\mathcal{N}=q^{\uparrow} q^{\downarrow}$ is the number operator defined in terms of the number $n_{q^{\uparrow}\left(q^{\downarrow}\right)}$ of $q^{\uparrow}\left(q^{\downarrow}\right)$ quarks.

\section{Chiral constituent quark model}

The QCD Lagrangian does not remain invariant under the chiral transformation $\left(\psi \rightarrow \gamma^{5} \psi\right)$ as the mass terms change sign as $\psi_{L} \rightarrow-\psi_{L}$ and $\psi_{R} \rightarrow \psi_{R}$. The Lagrangian will have global chiral symmetry of the $S U(3)_{L} \times S U(3)_{R}$ group if the mass terms are neglected. Around the scale of $1 \mathrm{GeV}$ the chiral symmetry is believed to be spontaneously broken to $S U(3)_{L+R}$. As a consequence, there exists a set of massless particles, referred to as the Goldstone bosons (GBs), which are identified with the observed ( $\pi, K, \eta$ mesons). Within the region of QCD confinement scale $\left(\Lambda_{Q C D} \simeq 0.1-0.3 \mathrm{GeV}\right)$ and the chiral symmetry breaking scale $\Lambda_{\chi S B}$, the constituent quarks, the octet of GBs ( $\pi, \mathrm{K}, \eta$ mesons), and the weakly interacting gluons are the appropriate degrees of freedom.

The effective Lagrangian describing interaction between quarks and a nonet of GBs, consisting of octet and a singlet, can now be expressed as

$$
\mathcal{L}_{\text {int }}=g_{8} \bar{\psi} \Phi \psi+g_{1} \bar{\psi} \frac{\eta^{\prime}}{\sqrt{3}} \psi=g_{8} \bar{\psi}\left(\Phi+\zeta \frac{\eta^{\prime}}{\sqrt{3}} I\right) \psi=g_{8} \bar{\psi}\left(\Phi^{\prime}\right) \psi,
$$

where $\zeta=g_{1} / g_{8}, g_{1}\left(g_{8}\right)$ is the coupling constant for the singlet (octet) GB and $I$ is the $3 \times 3$ identity matrix.

The basic idea in the $\chi \mathrm{CQM}[20]$ is the fluctuation process where the GBs are emitted by a constituent quark. These GBs further splits into a $q \bar{q}$ pair, for example, $q^{\uparrow(\downarrow)} \rightarrow \mathrm{GB}^{0}+q^{\prime \downarrow(\uparrow)} \rightarrow$ 
$(q q)^{0}+q^{\downarrow(\uparrow)}$, where $q q+q$ constitute the sea quarks [22, 23, 25]. The GB field can be expressed in terms of the GBs and their transition probabilities as

$$
\Phi^{\prime}=\left(\begin{array}{ccc}
\frac{\pi^{0}}{\sqrt{2}}+\beta \frac{\eta}{\sqrt{6}}+\zeta \frac{\eta^{\prime}}{\sqrt{3}} & \pi^{+} & \alpha K^{+} \\
\pi^{-} & -\frac{\pi^{0}}{\sqrt{2}}+\beta \frac{\eta}{\sqrt{6}}+\zeta \frac{\eta^{\prime}}{\sqrt{3}} & \alpha K^{o} \\
\alpha K^{-} & \alpha \bar{K}^{0} & -\beta \frac{2 \eta}{\sqrt{6}}+\zeta \frac{\eta^{\prime}}{\sqrt{3}}
\end{array}\right) .
$$

The transition probability of chiral fluctuation $u(d) \rightarrow d(u)+\pi^{+(-)}$, given in terms of the coupling constant for the octet GBs $\left|g_{8}\right|^{2}$, is defined as $a$ and is introduced by considering nondegenerate quark masses $M_{s}>M_{u, d}$. The probabilities of transitions of $u(d) \rightarrow s+K^{+(0)}, u(d, s) \rightarrow u(d, s)+\eta$, and $u(d, s) \rightarrow u(d, s)+\eta^{\prime}$ are given as $\alpha^{2} a, \beta^{2} a$ and $\zeta^{2} a$ respectively [22, 23]. The probability parameters $\alpha^{2} a$ and $\beta^{2} a$ are introduced by considering nondegenerate GB masses $M_{K}, M_{\eta}>M_{\pi}$ and the probability $\zeta^{2} a$ is introduced by considering $M_{\eta^{\prime}}>M_{K}, M_{\eta}$.

The sea quark flavor distribution functions can be calculated in $\chi \mathrm{CQM}$ by substituting for every valence (constituent) quark $q \rightarrow P_{q} q+|\psi(q)|^{2}$, where the transition probability of no emission of GB $P_{q}$ can be expressed in terms of the transition probability of the emission of a GB from any of the $u, d$, and $s$ quark. The spin structure of the nucleon after the inclusion of sea quarks generated through chiral fluctuation can be calculated by substituting for each valence (constituent) quark $q^{\uparrow \downarrow} \rightarrow$ $P_{q} q^{\uparrow \downarrow}+\left|\psi\left(q^{\uparrow \downarrow}\right)\right|^{2}$, where $\left|\psi\left(q^{\uparrow \downarrow}\right)\right|^{2}$ is the probability of transforming $q^{\uparrow \downarrow}$ quark after one interaction.

\section{Spin independent and spin dependent structure functions of the nucleon}

The nucleon structure is conventionally parameterized by the spin independent structure functions $F_{1}(x)$ and $F_{2}(x)$, and by the spin dependent structure functions $g_{1}(x)$ and $g_{2}(x)$, where $x$ is the Bjorken scaling variable. One useful probe of the nucleon spin structure is the longitudinal spin asymmetry $A_{1}(x)$. The scattering of a polarized lepton from a polarized proton can be used to measure the spin dependent structure function $g_{1}$ from the difference in cross sections for leptons with the same and unlike helicity as that of the proton. The longitudinal spin asymmetries can be defined as $A_{1}(x)=$ $\frac{\sigma^{\uparrow \uparrow}-\sigma^{\uparrow \downarrow}}{\sigma^{\uparrow \uparrow}+\sigma^{\uparrow \downarrow}} \simeq \frac{g_{1}(x)}{F_{2}(x)}$. The spin independent structure functions of the nucleon can be further defined in terms of the unpolarized distribution functions of the quarks as follows

$$
F_{1}^{N}(x)=\frac{1}{2} \sum_{u, d, s} e_{i}^{2}\left(q_{i}(x)+\bar{q}_{i}(x)\right), \quad F_{2}^{N}(x)=2 x F_{1}^{N}(x) .
$$

In the $\chi \mathrm{CQM}$, the unpolarized distribution function of the quarks can be defined in terms of the constituent or valence as well as the sea (antiquark) distribution functions as $q^{N}(x)=q_{\mathrm{V}}^{N}(x)+\bar{q}^{N}(x)$. Here we have the valence quark distribution functions for $p$ and $n$ as

$$
\int_{0}^{1} u_{\mathrm{V}}^{p}(x) d x=2, \int_{0}^{1} d_{\mathrm{V}}^{p}(x) d x=1, \int_{0}^{1} s_{\mathrm{V}}^{p}(x)\left(s_{\mathrm{V}}^{n}(x)\right) d x=0, \int_{0}^{1} u_{\mathrm{V}}^{n}(x) d x=1, \int_{0}^{1} d_{\mathrm{V}}^{n}(x) d x=2,
$$

and the sea quark distribution functions for $p$ and $n$ as

$$
\begin{aligned}
\bar{u}^{p}=\frac{a}{12}\left(21+\beta^{2}+4 \zeta+4 \zeta^{2}+\beta(2+4 \zeta)\right), & \bar{u}^{n} & =\frac{a}{12}\left(33+\beta^{2}-4 \zeta+4 \zeta^{2}+\beta(-2+4 \zeta)\right), \\
\bar{d}^{p}=\frac{a}{12}\left(33+\beta^{2}-4 \zeta+4 \zeta^{2}+\beta(-2+4 \zeta)\right), & \bar{d}^{n} & =\frac{a}{12}\left(21+\beta^{2}+4 \zeta+4 \zeta^{2}+\beta(2+4 \zeta)\right), \\
\bar{s}^{p}=3 a\left(\alpha^{2}+\frac{1}{9}(\beta-\zeta)^{2}\right), & \bar{s}^{n} & =3 a\left(\alpha^{2}+\frac{1}{9}(\beta-\zeta)^{2}\right) .
\end{aligned}
$$


There are no simple or straightforward rules which could allow incorporation of $x$ dependence in the valence quarks and the sea quarks. For the case of unpolarized valence quark distribution function, we have incorporated the $x$ dependence phenomenologically.

Using the unpolarized quark distribution functions, the structure function $F_{2}$ for the $p$ and $n \mathrm{Eq}$. (3) can be expressed as

$$
\begin{aligned}
& F_{2}^{p}(x)=\frac{4}{9} x\left(u_{\mathrm{V}}^{p}(x)+2 \bar{u}^{p}(x)\right)+\frac{1}{9} x\left(d_{\mathrm{V}}^{p}(x)+2 \bar{d}^{p}(x)+s_{\mathrm{V}}^{p}(x)+2 \bar{s}^{p}(x)\right), \\
& F_{2}^{n}(x)=\frac{4}{9} x\left(u_{\mathrm{V}}^{n}(x)+2 \bar{u}^{n}(x)\right)+\frac{1}{9} x\left(d_{\mathrm{V}}^{n}(x)+2 \bar{d}^{n}(x)+s_{\mathrm{V}}^{n}(x)+2 \bar{s}^{n}(x)\right) .
\end{aligned}
$$

The spin dependent structure function of the nucleon can similarly be defined in terms of the polarized distribution function of the quarks as

$$
g_{1}^{N}(x)=\frac{1}{2} \sum_{u, d, s} e_{i}^{2} \Delta q_{i}(x) .
$$

The polarized distribution function of the quarks can also be define in terms of polarized valence and sea quark distribution functions as $\Delta q^{N}(x)=\Delta q_{\mathrm{V}}^{N}(x)+\Delta q_{\mathrm{S}}^{N}$. Here we have the polarized valence quark distribution functions for $p$ and $n$ as

$$
\Delta u_{\mathrm{V}}^{p}=\frac{4}{3}, \quad \Delta d_{\mathrm{V}}^{p}=-\frac{1}{3}, \quad \Delta s_{\mathrm{V}}^{p}=0, \Delta u_{\mathrm{V}}^{n}=-\frac{1}{3}, \quad \Delta d_{\mathrm{V}}^{n}=\frac{4}{3}, \quad \Delta s_{\mathrm{V}}^{n}=0,
$$

and the polarized sea quark distribution functions for $p$ and $n$ as

$$
\begin{array}{rlrl}
\Delta u_{\mathrm{S}}^{p}=-\frac{a}{3}\left(7+4 \alpha^{2}+\frac{4}{3} \beta^{2}+\frac{8}{3} \zeta^{2}\right), & \Delta u_{\mathrm{S}}^{n} & =-\frac{a}{3}\left(2-\alpha^{2}-\frac{1}{3} \beta^{2}-\frac{2}{3} \zeta^{2}\right), \\
\Delta d_{\mathrm{S}}^{p}=-\frac{a}{3}\left(2-\alpha^{2}-\frac{1}{3} \beta^{2}-\frac{2}{3} \zeta^{2}\right), & \Delta d_{\mathrm{S}}^{n}=-\frac{a}{3}\left(7+4 \alpha^{2}+\frac{4}{3} \beta^{2}+\frac{8}{3} \zeta^{2}\right), \\
\Delta s_{\mathrm{S}}^{p}=-a \alpha^{2}, & \Delta s_{\mathrm{S}}^{n}=-a \alpha^{2} .
\end{array}
$$

Following Brodsky et al. [30], for the polarized valence quark distribution functions of $p$ and $n$ we have parametrized

$$
\begin{aligned}
& \Delta u_{\mathrm{V}}^{p}(x)=\Delta u_{\mathrm{V}}^{p}(1-x)^{3}, \quad \Delta d_{\mathrm{V}}^{p}(x)=\Delta d_{\mathrm{V}}^{p}(1-x)^{3}, \quad \Delta s_{\mathrm{V}}^{p}(x)=\Delta s_{\mathrm{V}}^{p}(1-x)^{3}, \\
& \Delta u_{\mathrm{V}}^{n}(x)=\Delta u_{\mathrm{V}}^{n}(1-x)^{3}, \quad \Delta d_{\mathrm{V}}^{n}(x)=\Delta d_{\mathrm{V}}^{n}(1-x)^{3}, \quad \Delta s_{\mathrm{V}}^{n}(x)=\Delta s_{\mathrm{V}}^{n}(1-x)^{3},
\end{aligned}
$$

and for the polarized sea quark distribution functions of $p$ and $n$ we have parametrized

$$
\begin{aligned}
& \Delta u_{\mathrm{S}}^{p}(x)=\Delta u_{\mathrm{S}}^{p}(1-x)^{5}, \quad \Delta d_{\mathrm{S}}^{p}(x)=\Delta d_{\mathrm{S}}^{p}(1-x)^{5}, \quad \Delta s_{\mathrm{S}}^{p}(x)=\Delta s_{\mathrm{S}}^{p}(1-x)^{5}, \\
& \Delta u_{\mathrm{S}}^{n}(x)=\Delta u_{\mathrm{S}}^{n}(1-x)^{5}, \quad \Delta d_{\mathrm{S}}^{n}(x)=\Delta d_{\mathrm{S}}^{n}(1-x)^{5}, \quad \Delta s_{\mathrm{S}}^{n}(x)=\Delta s_{\mathrm{S}}^{n}(1-x)^{5} .
\end{aligned}
$$

The structure function $g_{1}$ for $p$ and $n$ can respectively be calculated using the above equations and are expressed as

$$
g_{1}^{p}(x)=\frac{4}{9}\left(\Delta u^{p}\right)+\frac{1}{9}\left(\Delta d^{p}+\Delta s^{p}\right), \quad g_{1}^{n}(x)=\frac{4}{9}\left(\Delta u^{n}\right)+\frac{1}{9}\left(\Delta d^{n}+\Delta s^{n}\right) .
$$

After having formulated the $x$ dependence in the valence and sea quark distribution functions, we now consider the quantities which are measured at different $x$ and can expressed in terms of the above 
mentioned quark distribution functions. The proton and neutron longitudinal spin asymmetries are given by

$$
A_{1}^{p}(x)=\frac{4 \Delta u^{p}(x)+\Delta d^{p}(x)}{4 u^{p}(x)+d^{p}(x)}, \quad A_{1}^{n}(x)=\frac{4 \Delta u^{n}(x)+\Delta d^{n}(x)}{4 u^{n}(x)+d^{n}(x)} .
$$

These expressions can be rearranged to obtain the explicit ratio of polarized to unpolarized quark distribution functions for up and down quarks in the proton and neutron as

$$
\begin{aligned}
\frac{\Delta u^{p}(x)}{u^{p}(x)} & =\frac{4}{15} A_{1}^{p}(x)\left(4+\frac{d^{p}(x)}{u^{p}(x)}\right)-\frac{1}{15} A_{1}^{n}(x)\left(1+4 \frac{d^{p}(x)}{u^{p}(x)}\right) \\
\frac{\Delta d^{p}(x)}{d^{p}(x)} & =\frac{4}{15} A_{1}^{n}(x)\left(4+\frac{u^{p}(x)}{d^{p}(x)}\right)-\frac{1}{15} A_{1}^{p}(x)\left(1+4 \frac{u^{p}(x)}{d^{p}(x)}\right), \\
\frac{\Delta u^{n}(x)}{u^{n}(x)} & =\frac{4}{15} A_{1}^{p}(x)\left(1+4 \frac{d^{n}(x)}{u^{n}(x)}\right)-\frac{1}{15} A_{1}^{n}(x)\left(4+\frac{d^{p}(x)}{u^{p}(x)}\right), \\
\frac{\Delta d^{n}(x)}{d^{n}(x)} & =\frac{4}{15} A_{1}^{n}(x)\left(1+4 \frac{u^{n}(x)}{d^{n}(x)}\right)-\frac{1}{15} A_{1}^{p}(x)\left(4+\frac{u^{n}(x)}{d^{n}(x)}\right) .
\end{aligned}
$$

\section{Results and discussion}

In order to study the phenomenological quantities pertaining to the valence and sea quarks distribution functions and further compare the $\chi \mathrm{CQM}$ results with other model calculations and the available experimental data, we can study the $x$ dependence of the spin independent and spin dependent structure functions. To this end, we first fix the $\chi \mathrm{CQM}$ parameters which provide the basis to understand the extent to which the sea quarks contribute to the structure of the nucleon. The probabilities of fluctuations to pions, $K, \eta, \eta^{\prime}$ coming in the sea quark distribution functions are represented by $a, a \alpha^{2}, a \beta^{2}$, and $a \zeta^{2}$ respectively and can be obtained by taking into account strong physical considerations and carrying out a fine grained analysis using the well known experimentally measurable quantities pertaining to the spin and flavor distribution functions. The hierarchy for the probabilities, which scale as $\frac{1}{M_{q}^{2}}$, can be obtained as $a>a \alpha^{2} \geq a \beta^{2}>a \zeta^{2}$.
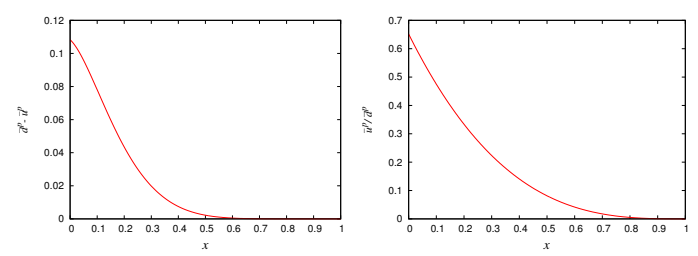

Figure 1. (color online). The sea quark flavor asymmetries for the case of $p: \bar{d}^{p}(x)-\bar{u}^{p}(x)$ and $\bar{u}^{p}(x) / \bar{d}^{p}(x)$ as a function of $x$ compared with the experimental data [9].

After having incorporated $x$ dependence in the valence and the sea quark distribution functions, we now discuss the variation of all the related phenomenological quantities in the range $0 \leq x \leq 1$. The valence quarks distribution functions of $p$ and $n$ vary as $u_{\mathrm{V}}^{p}(x)>d_{\mathrm{V}}^{p}(x)>s_{\mathrm{V}}^{p}(x)$, and $d_{\mathrm{V}}^{n}(x)>$ $u_{\mathrm{V}}^{n}(x)>s_{\mathrm{V}}^{n}(x)$. On the other hand, the sea quark distribution functions vary as $d_{\mathrm{S}}^{p}(x)>u_{\mathrm{S}}^{p}(x)>s_{\mathrm{S}}^{p}(x)$, and $u_{\mathrm{S}}^{n}(x)>d_{\mathrm{S}}^{n}(x)>s_{\mathrm{S}}^{n}(x)$. It is evident that there is $u$ quark dominance in the case of $p$ and $d$ quark dominance in the case of $n$. Since the total quark distribution functions are dominated by the 
valence quarks, the overall variation of the quark distribution functions is similar to the valence quark distribution functions. Even though the variation of sea quarks if different, but since the probability for the fluctuation of valence quarks to sea quarks depends upon the $\chi \mathrm{CQM}$ parameter $a$ and this probability of the occurrence of sea quarks cannot be more than 10-15\%. Therefore, $u^{p}(x)$ dominates in the case of $p$ and $d^{n}(x)$ dominates in the case of $n$. This observation can also be directly related to the measurement of the Gottfried integral for the case of nucleon which has shown a clear violation of GSR from $\frac{1}{3}$. The quark sea asymmetry $\int_{0}^{1}(\bar{d}(x)-\bar{u}(x)) d x$ which has been measured in the NMC and E866 experiments [8,9]. The NMC has reported $I_{G}^{p n}=\frac{1}{3}+\frac{2}{3}\left[\bar{u}^{p}-\bar{d}^{p}\right]=0.266 \pm 0.005[8]$ and the E866 has reported $I_{G}^{p n}=0.254 \pm 0.005$ [9]. A flavor symmetric sea $\left(\bar{u}^{N}=\bar{d}^{N}\right)$ would lead to $I_{G}^{p n}=\frac{1}{3}$. The $\chi \mathrm{CQM}$ result for the case of nucleon $\left(I_{G}^{p n}=0.254\right)$ is in good agreement with the available experimental data of E866 [9]. We have plotted some of the well known experimentally measurable quantities, for example, $\bar{d}^{p}(x)-\bar{u}^{p}(x)$ and $\bar{d}^{p}(x) / \bar{u}^{p}(x)$ in Fig. 1 . It is clear from the plots that when $x$ is small $\bar{d}^{p}(x)-\bar{u}^{p}(x)$ asymmetry is large implying the dominance of sea quarks in the low $x$ region. In fact, the sea quarks dominate only in the region where $x$ is smaller than 0.3 . At the values $x>0.3, \bar{d}-\bar{u}$ tends to 0 implying that there are no sea quarks in this region. To test the validity of the model as well as for the sake of completeness, we can present the results of our calculations for $\bar{d}^{p}(x)-\bar{u}^{p}(x)$ and $\bar{d}^{p}(x) / \bar{u}^{p}(x)$ whose data is available over a range of $x$ or at an average value of $x$. We find a good overall agreement with the data in these cases also. The data for $\bar{d}^{p}(x)-\bar{u}^{p}(x)$ is available for the ranges $x=0-1$ and $x=0.05-0.35$ and is given as $\int_{0}^{1}\left(\bar{d}^{p}(x)-\bar{u}^{p}(x)\right) d x=0.118 \pm 0.012$ and $\int_{0.05}^{0.35}\left(\bar{d}^{p}(x)-\bar{u}^{p}(x)\right) d x=0.0803 \pm 0.011$. We find that, in our model, $\int_{0}^{1}\left(\bar{d}^{p}(x)-\bar{u}^{p}(x)\right) d x=0.117$ and $\int_{0.05}^{0.35}\left(\bar{d}^{p}(x)-\bar{u}^{p}(x)\right) d x=0.08$ in these given $x$ ranges. The valence quark distribution however is spread over the entire $x$ region.
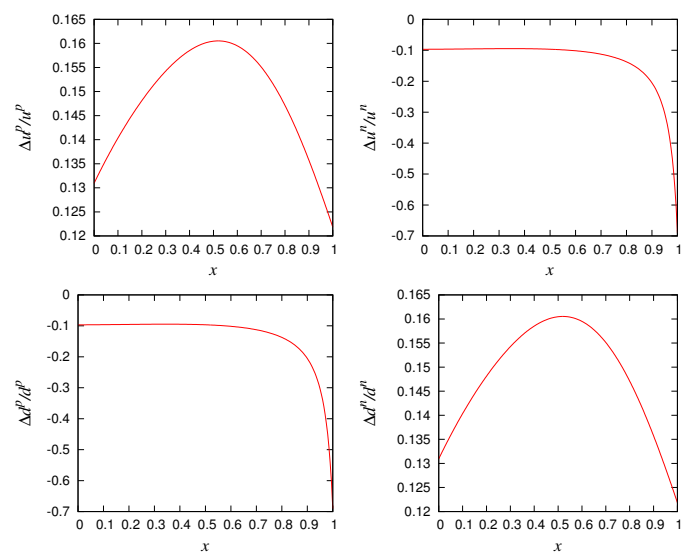

Figure 2. (color online). The ratio of polarized to unpolarized distribution functions for the $p$ and $n: \frac{\Delta u^{p}(x)}{u^{p}(x)}, \frac{\Delta u^{n}(x)}{u^{n}(x)}$, $\frac{\Delta d^{p}(x)}{d^{p}(x)}, \frac{\Delta d^{n}(x)}{d^{n}(x)}$ as a function of $x$.

In Fig. 2, the ratio of polarized to unpolarized quark distribution functions for up and down quarks in the $p$ and $n \frac{\Delta u^{p}(x)}{u^{p}(x)}, \frac{\Delta d^{p}(x)}{d^{p}(x)}$ and $\frac{\Delta u^{n}(x)}{u^{n}(x)}, \frac{\Delta d^{n}(x)}{d^{n}(x)}$ have been presented. It is clear from the figure that $\frac{\Delta d^{p}(x)}{d^{p}(x)}$ and $\frac{\Delta u^{n}(x)}{u^{n}(x)}$ show constant values at lower and higher $x$ and then suddenly fall off as $x \rightarrow 1$. This is unlike $\frac{\Delta u^{p}(x)}{u^{p}(x)}$ and $\frac{\Delta u^{n}(x)}{u^{n}(x)}$. The behavior of the unpolarized distribution functions of $u^{p}$ and $d^{p}$ is similar. 

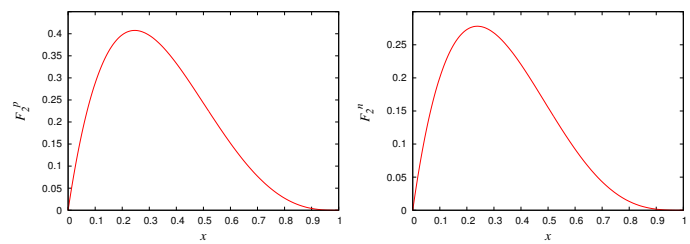

Figure 3. (color online). The spin independent structure functions for $p$ and $n: F_{2}^{p}(x)$ and $F_{2}^{n}(x)$.

They first rise at lower $x$ and then fall with $x \rightarrow 1$. However, the behavior of polarized distribution functions $\Delta u^{p}$ and $\Delta d^{p}$ is different. In this case, $\Delta u^{p}$ falls w.r.t $x$ in the positive direction whereas $\Delta d^{p}$ rises in the negative axis. In the $\frac{\Delta u^{p}(x)}{u^{p}(x)}$ graph, both the quantities in the numerator as well as the denominator are positive and fall with $x$ whereas in the $\frac{\Delta d^{p}(x)}{d^{p}(x)}$ graph the numerator is positive while the denominator is negative and rising. The results agree with the very recent analysis performed by the Jefferson Lab Angular Momentum (JAM) collaboration to produce a new parameterization [31] and the ratio $\frac{\Delta d^{p}(x)}{d^{p}(x)}$ was found to remain negative across all $x$. The NQM has the following predictions for the above mentioned quantities

$$
\frac{\Delta u^{p}(x)}{u^{p}(x)}=\frac{2}{3}, \quad \frac{\Delta d^{p}(x)}{d^{p}(x)}=-\frac{1}{3}, \quad \frac{\Delta u^{n}(x)}{u^{n}(x)}=-\frac{1}{3}, \quad \frac{\Delta d^{n}(x)}{d^{n}(x)}=\frac{2}{3} .
$$

Since $\Delta u$ and $\Delta d$ denote the difference between the quarks distributions polarized parallel and antiparallel to the polarized nucleon, the distribution when $x \rightarrow 1$ predicts that the structure functions should be dominated by valence quarks polarized parallel to the spin of the nucleon for the case of $\frac{\Delta u(x)}{u(x)}$ and by valence quarks polarized antiparallel to the spin of the nucleon for the case of $\frac{\Delta d(x)}{d(x)}$. Further, dramatically different behaviors for the $\frac{\Delta d(x)}{d(x)}$ ratio in different approaches allowed for $x \gtrsim 0.3$ highlights the critical need for precise data sensitive to the $d$ quark polarization at large $x$ values. Inclusion of nonzero orbital angular momentum could play an important role numerically. Further progress on this problem is expected with new data expected from several experiments at the $12 \mathrm{GeV}$ energy upgraded Jefferson Lab [32] which aim to measure polarization asymmetries of protons up to $x \sim 0.8$.

In Fig. 3, we have plotted the spin independent structure functions $F_{2}^{p}(x)$ and $F_{2}^{n}(x)$ for the case of $p$ and $n$. The plots clearly project out the distribution of the valence and sea quarks. The function has its peak at around $x \simeq 0.25$. Since the contribution of sea quarks decreases beyond this $x$, the function drops down to zero as $x \rightarrow 1$. There is no mechanism in NQM which can explain the contribution of sea quarks and it has the following predictions for the spin independent structure functions $F_{1}^{p}(x)$ and $F_{1}^{n}(x)$ at $x \rightarrow 1 F_{1}^{p}(x)=\frac{1}{2}$ and $F_{1}^{n}(x)=\frac{1}{6}$. These results may also be related to the Gottfried integral determined from $\frac{F_{2}^{p}(x)-F_{2}^{n}(x)}{2 x}$. The small $x$ part is suppressed relative to the NQM prediction. As $x \rightarrow 1$, the distribution is dominated by the valence quarks and sea quark asymmetry reduces to zero. This is a clean region to test the valence structure of the nucleon. Measurements of the spin independent structure function in the presently inaccessible low $\mathrm{x}$ region will provide crucial information on the low $x$ behavior of $F_{1}^{p}(x)$ and $F_{1}^{n}(x)$ and also allow access to the non-valence contribution in this region.

In Fig. 4, we have plotted the spin dependent structure functions $g_{1}^{p}(x)$ and $g_{1}^{n}(x)$ for the case of $p$ and $n$. For $g_{1}^{p}(x)$, we find that it constantly drops down to zero as $x$ increases beyond $x>0.5$ whereas for $g_{1}^{n}(x)$, it increases from -0.07 to 0 and again at $x>0.5$ it becomes zero. The NQM predicts $g_{1}^{p}(x)=\frac{5}{9}$ and $g_{1}^{n}(x)=0$. It is interesting to note that non-zero values of $g_{1}^{n}(x)$ for $x<0.5$ clearly implies the presence of sea quarks. Even though the valence quark distribution is spread over 

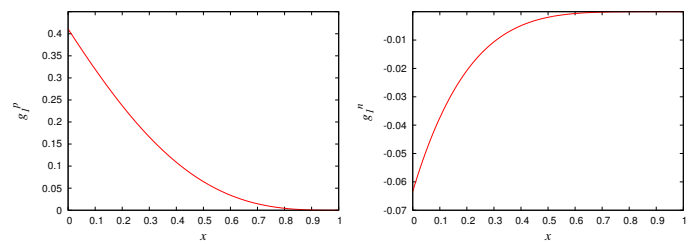

Figure 4. (color online). The spin dependent structure functions for $p$ and $n: g_{1}^{p}(x)$ and $g_{1}^{n}(x)$.
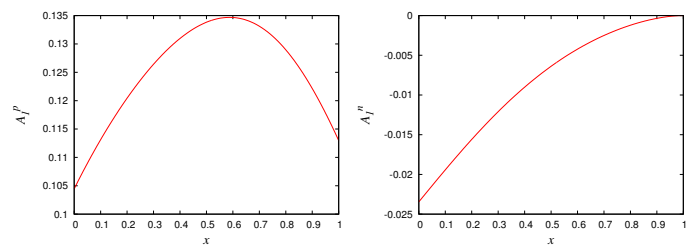

Figure 5. (color online). The longitudinal spin asymmetries for $p$ and $n: A_{1}^{p}(x)$ and $A_{1}^{n}(x)$ vs $x$.

the entire $x$ region and the sea quark distribution decreases with the increasing value of $x$, the valence and sea quarks are polarized in opposite direction and they mutually cancel the effect of each other at higher values of $x$. When compared with the data [5], we find that our results do not agree with the data at low values of $x$ but as the value of $x$ increases the results are more close.

In Fig. 5, the results for $A_{1}^{p}(x)$ and $A_{1}^{n}(x)$ have been presented. The NQM predictions for these quantities are $A_{1}^{p}(x)=\frac{5}{9}$ and $A_{1}^{n}(x)=0$. These results do not agree at all with the experimental results which show that $A_{1}^{p}(x)$ increases from 0 at $x \rightarrow 0$ to 1 at $x \rightarrow 1[3,33]$. However, the $A_{1}^{p}(x)$ in $\chi \mathrm{CQM}$ shows a peak at $x \simeq 0.5$. This low value of $A_{1}^{p}(x)$ at lower and higher values of $x$ be explained on the basis of the sea quarks as in the very low $x$ regime the sea quarks are not highly polarized and at large $x$ there are very few sea quarks and structure is dominated by the valence quarks. For the case of $A_{1}^{n}(x)$, the data [34] is negative at low $x$ and becomes positive at large $x$. In $\chi$ CQM, the results agree with the data at some values of $x$ and negative values are obtained. However, at large $x, A_{1}^{n}(x)$ continues to remain negative and becomes 0 only at $x \rightarrow 1$. This is because the $d$ quarks dominate in the valence structure of the $n$ and since they are negatively polarized they keep the values of $A_{1}^{n}(x)$ negative.

\section{ACKNOWLEDGMENTS}

H. D. would like to thank Department of Science and Technology (Ref No. SB/S2/HEP-004/2013), Government of India, for financial support.

\section{References}

[1] E.D. Bloom et al., Phys. Rev. Lett. 23, 930 (1969); M. Breidenbach et al., Phys. Rev. Lett. 23, 935 (1969).

[2] J. Ashman et al. (EMC Collaboration), Phys. Lett. B 206, 364 (1988); J. Ashman et al. (EMC Collaboration), Nucl. Phys. B 328, 1 (1989). 
[3] B. Adeva et al. (SMC Collaboration), Phys. Rev. D 58, 112001 (1998); B. Adeva et al. (SMC Collaboration), Phys. Rev. D 60, 072004 (1999).

[4] P. Adams et al., Phys. Rev. D 56, 5330 (1997); P.L. Anthony et al. (E142 Collaboration), Phys. Rev. Lett. 71, 959 (1993); K. Abe et al. (E143 Collaboration), Phys. Rev. Lett. 76, 587 (1996); K. Abe et al. (E154 Collaboration), Phys. Rev. Lett. 79, 26 (1997).

[5] A. Airapetian et al. (HERMES Collaboration), Phys. Rev. D 71, 012003 (2005); A. Airapetian et al. (HERMES Collaboration), Phys. Rev. D 75, 012007 (2007).

[6] C.A. Aidala et al., Rev. Mod. Phys. 85, 655 (2013).

[7] X. Zheng et al., Phys. Rev. C 70, 065207 (2004); D.S. Parno et al., Phys. Lett. B 744, 309 (2015).

[8] P. Amaudruz et al. (New Muon Collaboration), Phys. Rev. Lett. 66, 2712 (1991); M. Arneodo et al. (New Muon Collaboration), Phys. Rev. D 50, R1 (1994).

[9] E.A. Hawker et al. (E866/NuSea Collaboration), Phys. Rev. Lett. 80, 3715 (1998); J.C. Peng et al. (E866/NuSea Collaboration), Phys. Rev. D 58, 092004 (1998); R. S. Towell et al. (E866/NuSea Collaboration), ibid. 64, 052002 (2001).

[10] A. Baldit et al. (NA51 Collaboration), Phys. Lett. B 253, 252 (1994).

[11] K. Ackerstaff et al. (HERMES Collaboration), Phys. Rev. Lett. 81, 5519 (1998).

[12] K. Gottfried, Phys. Rev. Lett. 18, 1174 (1967).

[13] W.M. Alberico, S.M. Bilenky, and C. Maieron, Phys. Rept. 358, 227 (2002) ; U. Dore, Eur. Phys. J. H 37, 115 (2012).

[14] H. Abramowicz, J.G.H. de Groot, J. Knobloch, J. May, P. Palazzi, A. Para, F. Ranjard, and J. Rothberg et al., Z. Phys. C bf 15, 19 (1982); H. Abramowicz et al., Z. Phys. C 17, 283 (1983); Costa et al., Nucl. Phys. B 297, 244 (1988).

[15] A.O. Bazarko et al. (CCFR Collaboration and NuTeV Collaboration), Z. Phys C 65, 189 (1995).

[16] P. Vilain et al. (CHARM II Collaboration), Eur. Phys. J. C bf 11, 19 (1999).

[17] O. Samoylov et al. (NOMAD Collaboration), Nucl. Phys. B 876, 339 (2013).

[18] M. Goncharov et al. (NuTeV Collaboration), Phys. Rev. D 64, 112006 (2001); G.P. Zeller et al., Phys. Rev. Lett. 88, 091802 (2002); G.P. Zeller et al., Phys. Rev. D 65, 111103 (2002); D. Mason et al., Phys. Rev. Lett. 99, 192001 (2007).

[19] A. Kayis-Topaksu et al. (CHORUS Collaboration), Nucl. Phys. B 798, 1 (2008); A. KayisTopaksu et al., New J. Phys. 13, 093002 (2011).

[20] S. Weinberg, Physica A 96, 327 (1979); A. Manohar and H. Georgi, Nucl. Phys. B 234, 189 (1984).

[21] E.J. Eichten, I. Hinchliffe, and C. Quigg, Phys. Rev. D 45, 2269 (1992).

[22] T.P. Cheng and L.F. Li, Phys. Rev. Lett. 74, 2872 (1995); Phys. Rev. D 57, 344 (1998); Phys. Rev. Lett. 80, 2789 (1998).

[23] J. Linde, T. Ohlsson, and H. Snellman, Phys. Rev. D 57, 452 (1998); 57, 5916 (1998).

[24] X. Song, J.S. McCarthy, and H.J. Weber, Phys. Rev. D 55, 2624 (1997); X. Song, Phys. Rev. D 57, 4114 (1998).

[25] H. Dahiya and M. Gupta, Phys. Rev. D 64, 014013 (2001); H. Dahiya and M. Gupta, Phys. Rev. D 67, 074001 (2003); H. Dahiya and M. Gupta, Int. Jol. of Mod. Phys. A, Vol. 19, No. 29, 5027 (2004); H. Dahiya, M. Gupta and J.M.S. Rana, Int. Jol. of Mod. Phys. A, Vol. 21, No. 21, 4255 (2006); H. Dahiya and M. Gupta, Phys. Rev. D 78, 014001 (2008); N. Sharma, H. Dahiya, P.K. Chatley, and M. Gupta Phys. Rev. D 81, 073001 (2010); N. Sharma and H. Dahiya, Int. Jol. of Mod. Phys. A, Vol. 28, No. 14, 1350052 (2013); H. Dahiya and M. Randhawa, Phys. Rev. D 90, 074001 (2014); H. Dahiya, Phys. Rev. D 91, 094010 (2015); A. Girdhar, H. Dahiya and M. 
Randhawa, Phys. Rev. D 92, 033012 (2015).

[26] H. Dahiya and M. Gupta, Phys. Rev. D 66, 051501(R) (2002); H. Dahiya and M. Gupta, Phys. Rev. D 67, 114015 (2003).

[27] N. Sharma, H. Dahiya, P.K. Chatley, and M. Gupta, Phys. Rev. D 79, 077503 (2009); N. Sharma, H. Dahiya, and P.K. Chatley, Eur. Phys. J. A 44, 125 (2010).

[28] A.M. Torres, K.P. Khemchandani, N. Sharma, and H. Dahiya, Eur. Phys. Jol. A 48, 185 (2012); N. Sharma, A.M. Torres, K.P. Khemchandani, and H. Dahiya, Eur. Phys. Jol. A 49, 11 (2013).

[29] N. Sharma and H. Dahiya, Pramana, 81, 449 (2013); N. Sharma and H. Dahiya, Pramana, 80, 237 (2013).

[30] S.J Brodsky, M. Burkardt, and I. Schmidt, Nucl. Phys. B 441, 197 (1995); S.J Brodsky, and I. Schmidt, Phys. Lett. B 351, 344 (1995).

[31] P. Jimenez-Delgado, A. Accardi, W.Melnitchouk, Phys. Rev. D 89, 034025 (2014); P. JimenezDelgado, H. Avakian, W. Melnitchouk Phys. Lett. B 738, 263 (2014).

[32] Jefferson Lab experiments PR12-06-109, S. Kuhn et al.; PR12-06-110, J.-P. Chen et al.; PR1206-122, B. Wojtsekhowski et al., spokespersons.

[33] M.G. Alekseev et al. (COMPASS Collaboration), Phys. Lett. B 690, 466 (2010).

[34] D.S. Parno et al. (Jefferson Lab Hall A Collaboration), Phys. Lett. B 744, 309 (2015). 